\section{Violencia laboral en trabajadores del sector salud: revisión sistemática}

\author{
ANDREA PALMA $^{1, \mathrm{a}}$, ELISA ANSOLEAGA ${ }^{2, \mathrm{~b}, \mathrm{c}}$, MAGDALENA AHUMADA $^{\mathrm{c}}$
}

\section{Workplace violence among health care workers}

The aim of this review is to describe and to analyze scientific reports about occupational violence in the health area. A systematic review of the literature on Academic Search Complete (EBSCO Host), Medline, Pubmed, Scielo, Scopus and Web of Science was performed and 23 articles were selected for analysis. Most studies are quantitative, measuring workplace violence and its consequences, including multi-stratum samples. They mainly measure psychological and external violence and their subjects are hospital workers. Violence is conceived as coming from organizational dimensions. Facilitators of violence are related to the nature of the job and the most commonly studied consequences are related to mental health. The main consequences of the phenomenon are depressive symptoms, post-traumatic stress disorder (PTSD) and work stress. The nature of the job as a facilitator of violence relates primarily to the place of work and to the profession. Most of the studies are carried out with multi-stratum samples and combine workers from different services. However, studies in psychiatric services and with nursing staff are also common.

(Rev Med Chile 2018; 146: 213-222)

Key words: Mental Health; Occupational Health; Stress Disorders, Post-traumatic; Workplace Violence.
'Programa de Doctorado en

Psicología, Facultad de Psicología, Universidad Diego Portales.

Santiago, Chile.

2Programa de Estudios

Psicosociales del Trabajo, Facultad de Psicología, Universidad Diego

Portales. Santiago, Chile.

aTrabajadora Social.

bPhD en Salud Pública.

'Psicóloga.

Fuente de apoyo financiero: Proyecto FONDECYT Regular 2017 No 1170239: "Prevalencia, formas de manifestación y factores facilitadores de la violencia laboral y sus efectos en la salud mental en población asalariada en Chile atendiendo desigualdades sociales y de género".

Recibido el 26 de mayo de 2017 , aceptado el 7 de noviembre de 2017.

Correspondencia a: Andrea Palma Facultad de psicología UDP Vergara 275, Santiago, Chile. Teléfono: 82702376 anpalma2004@yahoo.es
E n Chile, tanto el Código de Buenas Prácticas Laborales para la Administración Central del Estado como el Instructivo Presidencial sobre Buenas Prácticas Laborales en el Desarrollo de Personas en el Estado, exigen abordar la temática del buen trato en los hospitales, sin embargo, existe escaso conocimiento respecto al modo de hacerlo.

El concepto de buen trato se inscribe en el ámbito de la violencia laboral. Existen diferentes definiciones de violencia laboral (en adelante VL), la Organización Internacional del Trabajo (OIT) propone que la VL incluye a todos los comportamientos no razonables con los que se perjudica a alguien en el contexto laboral ${ }^{1}$. Asimismo, la VL se clasifica según el foco de análisis: tipo de agresión (física, psicológica y/o sexual) y según el perpetrador (violencia interna, si se trata de un miembro de la organización y externa cuando es un usuario o los acompañantes de éste).

El estudio de la VL tiene larga data y ha avanzado desde modelos que ponen énfasis en aspectos individuales de las víctimas y victimarios hacia miradas macrosociales. Einarsen $(2000)^{2}$ propuso clasificar los estudios del tema en tres enfoques: los que enfatizan en las características de personalidad de víctimas y perpetradores; los que focalizan en la relación interpersonal entre los involucrados; y los que se centran en las características de la organización del trabajo. Además, los estudios sobre VL han analizado el riesgo de exposición que tienen diferentes trabajadores, señalando que el riesgo de exposición a violencia en el trabajo es particularmente alto en puestos de trabajo que implican prestar servicios a personas ${ }^{3}$. Así, el sector salud destaca por la naturaleza del trabajo como 
un facilitador importante para el surgimiento de $\mathrm{VL}^{4,6-10}$

La relevancia social de la VL radica en la tendencia generalizada al alza ${ }^{11}$ y las graves consecuencias que tiene a nivel personal y organizacional. El propósito de este trabajo es visibilizar la VL como un asunto relevante del trabajo en salud mediante el análisis de la producción científica puesto que, si bien la importancia del buen trato laboral en el sector es conocido, es un asunto escasamente investigado $y$, en consecuencia, las respuestas para enfrentarlo son más intuitivas que basadas en la evidencia.

Dentro de las consecuencias a nivel personal se han observado consecuencias relevantes para la salud mental tales como: trastorno de estrés postraumático (TEPT) ${ }^{12}$, uso de antidepresivos y ansiolíticos ${ }^{13}$, depresión ${ }^{14,15}$, estrés laboral ${ }^{5,9}$, empeoramiento de la salud mental ${ }^{16-18}$, angustia relacionada con el trabajo ${ }^{5,19}$, afectividad negativa $^{18}$, irritabilidad y síntomas somáticos ${ }^{19}$, ira, decepción, humillación, ansiedad, miedo, angustia, sensación de impotencia, aislamiento y sentimiento de culpa ${ }^{6}$. En las consecuencias organizacionales se encuentran: aumento de las licencias médicas; disminución de la eficacia laboral y la participación en el proceso de trabajo y toma de decisiones; deterioro de la relación con los pacientes; rotación laboral $\mathrm{y}$, aumento del abandono de la profesión ${ }^{20,21}$.

Teniendo en cuenta la evidencia existente sobre los efectos a nivel personal y organizacional de la VL en el sector salud, es relevante analizar la evidencia científica disponible lo que contribuye a una mayor comprensión del problema. Para estos efectos, se realizó una revisión sistemática de la literatura ${ }^{22}$.

\section{Metodología y Materiales}

La revisión sistemática consiste en un "diseño de investigación observacional y retrospectivo, que sintetiza los resultados de múltiples investigaciones primarias" 22 . Ella se llevó a cabo con análisis de contenido consistente en "una técnica de investigación para la descripción objetiva, sistemática y cuantitativa del contenido manifiesto de las comunicaciones, que tiene como primer objetivo interpretarlas" ${ }^{23}$ donde, tal como lo recomienda esta técnica, se establecieron subcategorías a partir de variables preestablecidas con las que se levantó la información de la literatura seleccionada. Posteriormente, se realizó una síntesis de la distribución de las subcategorías mediante técnicas cuantitativas.

En una primera etapa se rastrearon individualmente términos relativos a violencia en el trabajo: "WorkplaceViolence"; "WorkplaceHarassment"; "OccupationalViolence"; "Workviolence"; "Mobbing" e "Incivility", tanto en inglés como en español. Posteriormente se buscaron los términos antes señalados con palabras relativas a salud mental: "work stress"; "mental health"; "distress"; "mental disorder" y "mental depression", tanto en inglés como en español. Finalmente, se añadieron términos propios del trabajo en salud: "HealthServices"; "HealthWorkers" y "CareWorkers", tanto en inglés como en español.

La búsqueda de la literatura incluyó las bases de datos: Web of Science, AcademicSearch Complete (EBSCO Host), Medline, PubMed, Scielo y Scopus. Se buscó en idioma inglés y español en todos los buscadores a excepción de Medline pues sólo ofrece en inglés. Los términos de búsqueda fueron rastreados en el abstract para los buscadores AcademicSearch Complete (EBSCO Host), Medline, Scielo, Pubmed y Scopus. Mientras que en Web of Science fueron buscados en Tema.

Los criterios de inclusión fueron: estudios empíricos que hacen referencia a VL en contextos laborales de salud, publicados entre los años 2011 a 2015 -puesto que es un estándar en términos de actualización de la literatura considerar estudios de no más de 5 a 10 años de antigüedad ${ }^{24}$-, escritos en inglés o español, publicados en revistas indexadas en ISI, Scopus o Scielo. Los criterios de exclusión fueron: artículos no empíricos, otras poblaciones, publicaciones no indexadas, artículos no disponibles.

La Figura 1 grafica el proceso de búsqueda, selección, inclusión y exclusión de los registros. Al terminar el proceso se seleccionaron 23 artículos para la revisión (Tabla 1).

Para la revisión de los artículos seleccionados se construyó una base de datos en la que se incluyeron 16 categorías (Tabla 2).

\section{Resultados}

En el período analizado (2011 a 2015) existe un alza en las publicaciones, especialmente en 2013 y 2015. EE. UU. $(21,7 \%)$, Italia $(17,4 \%)$ y UK $(13 \%)$ 


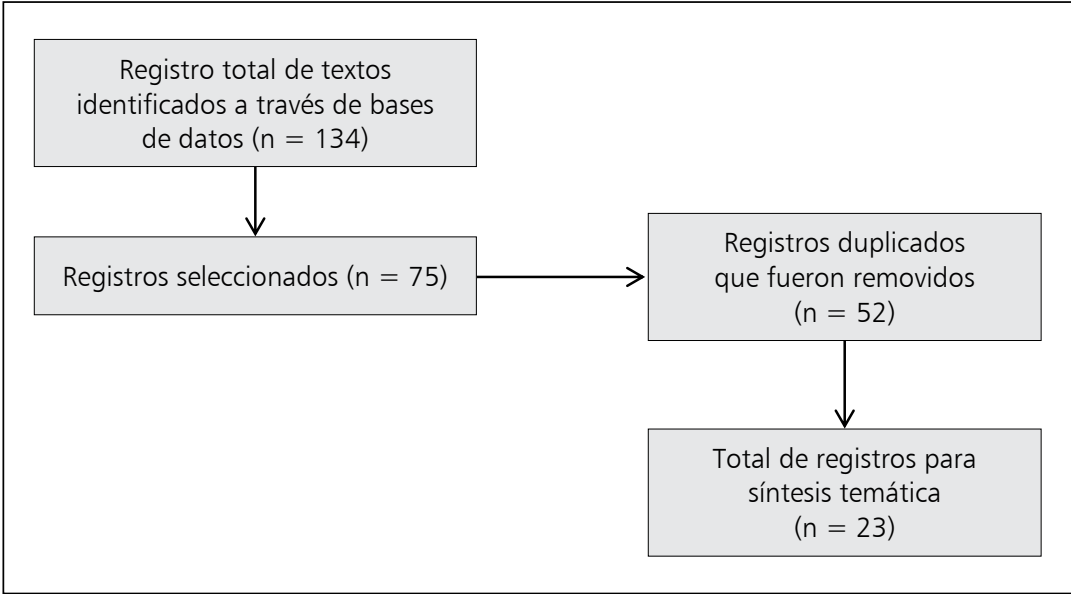

Figura 1. Proceso de búsqueda, selección, inclusión y exclusión de los registros.

Tabla 1. Artículos incorporados en la revisión

\begin{tabular}{|c|c|c|c|}
\hline Autores & Año & Título & Revista \\
\hline $\begin{array}{l}\text { Acosta M., Torres T., Díaz } \\
\text { D., Aguilera M. y Pozos B. }\end{array}$ & 2013 & $\begin{array}{l}\text { Seguro Popular, condiciones psicosociales de trabajo y } \\
\text { violencia en empleados de una institución de salud en } \\
\text { México: un análisis desde el modelo de los determinantes } \\
\text { sociales de salud }\end{array}$ & $\begin{array}{l}\text { Rev. Fac. Nac. Salud } \\
\text { Pública }\end{array}$ \\
\hline $\begin{array}{l}\text { Ariza-Montes A., Muniz } \\
\text { NM., Montero-Simo MJ. \& } \\
\text { Araque-Padilla RA. }\end{array}$ & 2013 & Workplace Bullying among Healthcare Workers & $\begin{array}{l}\text { International Journal } \\
\text { of Environmental } \\
\text { Research and Public } \\
\text { Health }\end{array}$ \\
\hline $\begin{array}{l}\text { Arnetz J., Aranyos D., Ager } \\
\text { J. y Upfal M. }\end{array}$ & 2011 & $\begin{array}{l}\text { Development and Application of a Population-Based } \\
\text { System for Workplace Violence Surveillance in Hospitals }\end{array}$ & $\begin{array}{l}\text { American Journal of } \\
\text { Industrial Medicine }\end{array}$ \\
\hline Baby M, Glue P. y Carlyle D. & 2014 & $\begin{array}{l}\text { 'Violence is Not Part of Our Job': A Thematic Analysis of } \\
\text { Psychiatric Mental Health Nurses' Experiences of Patient } \\
\text { Assaults from a New Zealand Perspective }\end{array}$ & $\begin{array}{l}\text { Issues in Mental } \\
\text { Health Nursing }\end{array}$ \\
\hline $\begin{array}{l}\text { Bernaldo-De-Quirós M., } \\
\text { Piccini A., Gómez M. y } \\
\text { Cerdeira J. }\end{array}$ & 2015 & $\begin{array}{l}\text { Psychological consequences of aggression in pre-hospital } \\
\text { emergency care: Cross sectional survey }\end{array}$ & $\begin{array}{l}\text { International Journal } \\
\text { of Nursing Studies }\end{array}$ \\
\hline $\begin{array}{l}\text { Carter M., Thompson N., } \\
\text { Crampton P., Morrow G., } \\
\text { Burford B., Gray C. y Illing J. }\end{array}$ & 2013 & $\begin{array}{l}\text { Workplace bullying in the UK NHS: A questionnaire and } \\
\text { interview study on prevalence, impact and barriers to } \\
\text { reporting }\end{array}$ & BMJ Open \\
\hline $\begin{array}{l}\text { Cavanaugh, C., Campbell J. } \\
\text { y Messing J. }\end{array}$ & 2014 & $\begin{array}{l}\text { A Longitudinal Study of the Impact of Cumulative Vio- } \\
\text { lence Victimization on Comorbid Posttraumatic Stress and } \\
\text { Depression Among Female Nurses and Nursing Personnel }\end{array}$ & $\begin{array}{l}\text { Workplace Health \& } \\
\text { Safety }\end{array}$ \\
\hline $\begin{array}{l}\text { Dement J., Lipscomb H., } \\
\text { Schoenfisch A. y Pompeii L. }\end{array}$ & 2014 & $\begin{array}{l}\text { Impact of hospital Type II violent events: Use of psycho- } \\
\text { tropic drugs and mental health services. }\end{array}$ & $\begin{array}{l}\text { American Journal of } \\
\text { Industrial Medicine }\end{array}$ \\
\hline $\begin{array}{l}\text { Imran N, Pervez M, Farooq } \\
\text { R. y Asghar A. }\end{array}$ & 2013 & $\begin{array}{l}\text { Aggression and violence towards medical doctors and } \\
\text { nurses in a public health care facility in Lahore, Pakistan: } \\
\text { a preliminary investigation. }\end{array}$ & $\begin{array}{l}\text { Khyber Medical } \\
\text { University Journal }\end{array}$ \\
\hline $\begin{array}{l}\text { Itzhaki M., Peles-Bortz A., } \\
\text { Kostistky H., Barnoy D., } \\
\text { Filshtinsky V. y Bluvstein I. }\end{array}$ & 2015 & $\begin{array}{l}\text { Exposure of mental health nurses to violence associat- } \\
\text { ed with job stress, life satisfaction, staff resilience, and } \\
\text { post-traumatic growth }\end{array}$ & $\begin{array}{l}\text { International Journal } \\
\text { of Mental Health } \\
\text { Nursing }\end{array}$ \\
\hline
\end{tabular}


Tabla 1. Artículos incorporados en la revisión (continuación)

\begin{tabular}{|c|c|c|c|}
\hline Autores & Año & Título & Revista \\
\hline $\begin{array}{l}\text { Leiter M., Laschinger H., } \\
\text { Day A. y Oore D. }\end{array}$ & 2011 & $\begin{array}{l}\text { The Impact of Civility Interventions on Employee Social } \\
\text { Behavior, Distress, and Attitudes }\end{array}$ & $\begin{array}{l}\text { Journal of Applied } \\
\text { Psychology }\end{array}$ \\
\hline Magnavita N. & 2014 & $\begin{array}{l}\text { Workplace Violence and Occupational Stress in Healthcare } \\
\text { Workers: A Chicken-and-Egg Situation Results of a 6-Year } \\
\text { Follow-up Study }\end{array}$ & $\begin{array}{l}\text { Journal of Nursing } \\
\text { Scholarship }\end{array}$ \\
\hline Magnavita N. y Fileni A. & 2012 & Violence against radiologists. II: psychosocial factors & Radiología Médica \\
\hline $\begin{array}{l}\text { Magnavita N., Fileni A., } \\
\text { Pescarini L. y Magnavita G. }\end{array}$ & 2012 & $\begin{array}{l}\text { Violence against radiologists. I: prevalence and preventive } \\
\text { measures }\end{array}$ & Radiología Médica \\
\hline $\begin{array}{l}\text { Magnavita N. y } \\
\text { Heponiemi T. }\end{array}$ & 2012 & $\begin{array}{l}\text { Violence towards health care workers in a Public Health } \\
\text { Care Facility in Italy: a repeated cross-sectional study }\end{array}$ & $\begin{array}{l}\text { Bmc Health Services } \\
\text { Research }\end{array}$ \\
\hline $\begin{array}{l}\text { Picakciefe M., Acar G., } \\
\text { Colak Z. y Kilic I. }\end{array}$ & 2015 & $\begin{array}{l}\text { The relationship between sociodemographic characteris- } \\
\text { tics, work conditions, and level of "Mobbing" of Health } \\
\text { Workers in Primary Health Care }\end{array}$ & J Interpers Violence \\
\hline $\begin{array}{l}\text { Ridenour M., Lanza M., } \\
\text { Hendricks S., Hartley D., } \\
\text { Rierdan J., Zeiss R. y } \\
\text { Amandus H. }\end{array}$ & 2015 & $\begin{array}{l}\text { Incidence and risk factors of workplace violence on psy- } \\
\text { chiatric staff }\end{array}$ & $\begin{array}{l}\text { Work-a Journal } \\
\text { of Prevention } \\
\text { Assessment \& } \\
\text { Rehabilitation }\end{array}$ \\
\hline Rodwell J. y Demir D. & 2012 & $\begin{array}{l}\text { Psychological consequences of bullying for hospital and } \\
\text { aged care nurses }\end{array}$ & $\begin{array}{l}\text { International Nursing } \\
\text { Review }\end{array}$ \\
\hline $\begin{array}{l}\text { Sabri B., St Vil N., } \\
\text { Campbell J., Fitzgerald S., } \\
\text { Kub J. y Agnew J. }\end{array}$ & 2015 & $\begin{array}{l}\text { Racial and Ethnic Differences in Factors Related to Work- } \\
\text { place Violence Victimization }\end{array}$ & $\begin{array}{l}\text { Western Journal of } \\
\text { Nursing Research }\end{array}$ \\
\hline $\begin{array}{l}\text { Travetto C., Daciuk N., } \\
\text { Fernández S., Ortiz P., } \\
\text { Mastandueno R., Prats M., } \\
\text { et al. }\end{array}$ & 2015 & Agresiones hacia profesionales en el ámbito de la salud & $\begin{array}{l}\text { Revista Panamericana } \\
\text { de Salud Pública }\end{array}$ \\
\hline $\begin{array}{l}\text { Wood S., Braeken J. y } \\
\text { Niven K. }\end{array}$ & 2013 & $\begin{array}{l}\text { Discrimination and Well-Being in Organizations: Testing } \\
\text { the Differential Power and Organizational Justice Theories } \\
\text { of Workplace Aggression }\end{array}$ & $\begin{array}{l}\text { Journal of Business } \\
\text { Ethics }\end{array}$ \\
\hline $\begin{array}{l}\text { Wooster L., Farnham F. y } \\
\text { James D. }\end{array}$ & 2013 & $\begin{array}{l}\text { The prevalence of stalking, harassment and aggressive/ } \\
\text { intrusive behaviours towards general practitioners }\end{array}$ & $\begin{array}{l}\text { Journal of Forensic } \\
\text { Psychiatry \& } \\
\text { Psychology }\end{array}$ \\
\hline $\begin{array}{l}\text { Yada H., Abe H., Omori H., } \\
\text { Matsuo H., Masaki O., } \\
\text { Ishida Y. y Katoh T. }\end{array}$ & 2014 & $\begin{array}{l}\text { Differences in job stress experienced by female and male } \\
\text { Japanese psychiatric nurses }\end{array}$ & $\begin{array}{l}\text { International Journal } \\
\text { of Mental Health } \\
\text { Nursing }\end{array}$ \\
\hline
\end{tabular}

concentran la mayor producción académica. Latinoamérica está escasamente representada con sólo dos estudios.

Los artículos seleccionados provienen de: Web of Science $(78,3 \%)$, Scielo $(8,7 \%)$, en PubMed, EBSCO y Scopus se seleccionó un artículo de cada uno. El 91,3\% $(n=21)$ de los artículos está en idioma inglés y los dos restantes en español, lo que podría constituir una dificultad en el acceso a estudios realizados en Latinoamérica. La mayoría de los estudios son empíricos y utilizan metodologías cuantitativas (87\%).

La mayoría de los estudios utilizó una muestra multiestamental $(47,8 \%)$, seguidos por aquellos con personal de enfermería (30,4\%), los efectuados sólo con médicos (13\%) y los realizados en otros profesionales de la salud, sin incluir más estamentos (8,7\%) (Tabla 3). Veinte estudios fueron realizados en Hospitales (87\%), dos en Atención Primaria $(8,7 \%)$ y uno mixto $(4,3 \%)$. 
Tabla 2. Definición de categorías de análisis

\begin{tabular}{|c|c|}
\hline $\begin{array}{l}\text { Nombre de la } \\
\text { categoría }\end{array}$ & Definición \\
\hline Título & Título del artículo analizado \\
\hline Año & Año de publicación \\
\hline Revista & Revista en que se publicó el estudio \\
\hline Base de datos & Base de datos de la que se seleccionó el artículo \\
\hline Idioma & Idioma del artículo \\
\hline Objetivo & Principal objetivo del artículo \\
\hline Abstract & Resumen del estudio \\
\hline Metodología & Se clasificó en: cuantitativa, cualitativa o mixta \\
\hline Muestra & Tipo de muestreo, tamaño y área laboral \\
\hline Instrumentos & Se clasificó entre aquellos que miden violencia laboral y los que miden otras variables \\
\hline $\begin{array}{l}\text { Definición de } \\
\text { violencia }\end{array}$ & $\begin{array}{l}\text { Concepto de violencia laboral utilizado en el estudio. Si se inscribe dentro definiciones que la con- } \\
\text { sideran un fenómeno individual, interaccional u organizacional }\end{array}$ \\
\hline Otras variables & $\begin{array}{l}\text { Otras variables principales incluidas en el estudio. Si se trata de variables laborales, de salud, sociales } \\
\text { o de afrontamiento del fenómeno }\end{array}$ \\
\hline $\begin{array}{l}\text { Tipo de violencia } \\
\text { laboral }\end{array}$ & $\begin{array}{l}\text { Tipos de violencia laboral incluidos en el estudio. Según tipo de agresión (física, psicológica, sexual) } \\
\text { y según procedencia (interna o externa) }\end{array}$ \\
\hline $\begin{array}{l}\text { Facilitadores de la } \\
\text { violencia laboral }\end{array}$ & $\begin{array}{l}\text { Factores mencionados en el estudio como contribuyentes a que aparezca o se mantenga el fenó- } \\
\text { meno }\end{array}$ \\
\hline $\begin{array}{l}\text { Consecuencias de la } \\
\text { violencia laboral }\end{array}$ & $\begin{array}{l}\text { Consecuencias personales y organizacionales de la violencia laboral. Para las primeras, si se trata } \\
\text { de consecuencias de salud, laborales o familiares. Para las segundas, se realizó categorización } \\
\text { emergente }\end{array}$ \\
\hline $\begin{array}{l}\text { Afrontamiento de la } \\
\text { violencia laboral }\end{array}$ & $\begin{array}{l}\text { Modos de afrontar la violencia laboral mencionados en el estudio. Si se trata de modos de afronta- } \\
\text { miento a nivel personal u organizacional }\end{array}$ \\
\hline
\end{tabular}

Tabla 3. Distribución muestral según estamento

\begin{tabular}{|lcr|}
\hline Estamento & \multicolumn{2}{c|}{ n artículos } \\
\hline Multiestamental & 11 & $(47,8 \%)$ \\
\hline Personal de enfermería & 7 & $(30,4 \%)$ \\
Médicos & 3 & $(13,0 \%)$ \\
Otros profesionales de la salud & 2 & $(8,7 \%)$ \\
\hline
\end{tabular}

La distribución de los estudios respecto del lugar de trabajo de la muestra es la siguiente: cuatro se efectuaron con trabajadores de salud mental y psiquiatría $(17,4 \%)$, dos con trabajadores de servicios de cuidados de adultos mayores $(8,7 \%)$, dos con médicos de radiología $(8,7 \%)$, uno con personal de urgencia $(4,3 \%)$ y 14 con funcionarios de diversos servicios $(60,9 \%)$.
Los instrumentos más utilizados para medir VL fueron el NegativeActsQuestionnaire NAQ- ${ }^{25}$, la versión italiana de la Forma de Incidentes Violen$\operatorname{tos}^{26}$ y el reporte de incidentes en la Base de datos OSHA $^{27}$, encontrándose cada uno de ellos en tres artículos ( $13 \%$ cada uno). Los artículos restantes $(60,9 \%)$ utilizan catorce instrumentos diferentes para medir VL. En la mayoría de los artículos $(60,9 \% ; n=14)$ se concibe la violencia como proveniente de dimensiones organizacionales (Tabla 4).

Respecto al tipo de violencia estudiado, la mayoría de los artículos hacen referencia a violencia física y psicológica $(56,5 \% ; \mathrm{n}=13)$ y 6 estudian exclusivamente violencia psicológica $(26,1 \%)$ (Tabla 5). En relación al tipo de violencia según su procedencia, la mayor parte de los artículos estudian violencia externa $(39,1 \%)$ y 7 hacen referencia a violencia interna y externa $(30,4 \%)$ (Tabla 6$)$. 
Tabla 4. Concepto de violencia laboral como fenómeno individual, interaccional u organizacional

\begin{tabular}{|lrr|}
\hline Concepto de violencia & n artículos \\
\hline Basado en dimensiones organizacionales & 14 & $(60,9 \%)$ \\
\hline Producto de la interacción & 4 & $(17,4 \%)$ \\
\hline Consecuencia de características individuales & 2 & $(8,7 \%)$ \\
\hline Más de un tipo & 3 & $(13 \%)$ \\
\hline
\end{tabular}

Tabla 5. Tipos de violencia según forma de agresión

\begin{tabular}{|lcr|}
\hline Tipo de violencia & \multicolumn{2}{c|}{ n artículos } \\
\hline Física y psicológica & 13 & $(56,5 \%)$ \\
\hline Psicológica & 6 & $(26,1 \%)$ \\
\hline Física, psicológica y sexual & 4 & $(17,4 \%)$ \\
\hline
\end{tabular}

Tabla 6. Tipos de violencia según procedencia

\begin{tabular}{|llr|}
\hline Tipo de violencia & \multicolumn{2}{c|}{ n artículos } \\
\hline Externa & 9 & $(39,1 \%)$ \\
\hline Interna y externa & 7 & $(30,4 \%)$ \\
\hline Interna & 6 & $(26,1 \%)$ \\
No es posible identificar & 1 & $(4,4 \%)$ \\
\hline
\end{tabular}

En relación a las covariables de la VL, 43,3\% de ellas corresponde a variables laborales, siendo las más utilizadas la satisfacción en el trabajo $(n=3)$ y la ocupación $(\mathrm{n}=3)$. El $41 \%$ fueron variables de salud, donde el estrés laboral $(\mathrm{n}=4)$, la depresión $(\mathrm{n}=3)$ y la percepción de salud mental general $(\mathrm{n}=3)$ fueron las más medidas. El 10,4\% corresponde a variables sociales, y $4,5 \%$ a variables de afrontamiento de VL.

Los facilitadores de VL que aparecen más comúnmente en los estudios son la naturaleza del trabajo $(47,8 \%)$ y los riesgos psicosociales laborales que son mencionados en $39,1 \%$ de los artículos revisados.

Los estudios revisados reportan que las consecuencias más comunes de la violencia a nivel personal se observan en la salud mental $(73,9 \%$ de las investigaciones lo mencionan) y en el ámbito laboral (52,1\% de los estudios).
Respecto a las consecuencias organizacionales de la VL, se menciona la disminución de la calidad de la atención $(\mathrm{n}=10,43,5 \%)$ y la disminución de productividad $(\mathrm{n}=9: 39,1 \%)$.

Los mecanismos individuales para afrontar la violencia aparecen en $34,8 \%$ de los artículos $(\mathrm{n}=8)$. En los mecanismos organizaciones $(43,5 \%)$ destaca el apoyo social $(13 \% ; \mathrm{n}=3)$ y el entrenamiento para enfrentar el fenómeno $(8,7 \% ; n=2)$.

En relación a los resultados de los artículos destaca en primer lugar, que los funcionarios de psiquiatría son más propensos a sufrir violencia física y verbal en comparación con los funcionarios de otros servicios clínicos ${ }^{8,28}$, en segundo lugar, el personal de enfermería es el que presenta mayor prevalencia del fenómeno a nivel psicológico y verbal, fluctuando entre $24,6 \%{ }^{29}$ hasta $89 \%{ }^{28}$.

Finalmente, no hay coincidencia respecto a los perpetradores más comunes, existiendo una gran fluctuación en la procedencia de la VL. Desde $15 \%{ }^{30}$ hasta $68,3 \%{ }^{10}$ sería efectuada por pacientes; y desde $23 \%$ en promedio para agresiones físicas y verbales ${ }^{10}$ hasta $75,2 \%$ para agresión verbal sería por parte de familiares ${ }^{28}$. Respecto a la violencia interna existe gran variabilidad en la forma de medición, por lo que resulta complejo un pronunciamiento respecto a las prevalencias reportadas en los artículos.

\section{Discusión}

La revisión de la literatura indicó un aumento de publicaciones respecto a la VL en el sector de salud en relación a años anteriores, lo que se condice con revisiones previas ${ }^{3,4}$, demostrando un notorio aumento de la investigación en VL en las últimas dos décadas. Esto puede relacionarse con el aumento del fenómeno a nivel mundial que constatan otras revisiones ${ }^{20} \mathrm{o}$ bien con el interés que ha despertado en el campo de la investigación.

Este estudio coincide con revisiones previas ${ }^{21,31}$ respecto a los objetivos y métodos: medir la tasa de VL, las consecuencias y los factores facilitadores. Asimismo, son consecuencias relevantes de la VL la sintomatología depresiva y de TEPT, añadiéndose en esta revisión el estrés laboral ${ }^{21}$. En relación a los factores facilitadores, revisiones previas establecieron como centrales la escasez de programas de capacitación sobre VL, ausencia 
de protocolos de gestión ${ }^{20}$ y el género de las víctimas $^{31-33}$. En cambio, en esta revisión emergen como facilitadores centrales: la naturaleza de la labor, trabajar en urgencias y psiquiatría supone mayor riesgo de sufrir violencia. Asimismo, pertenecer al estamento de enfermería, la falta de experiencia y las deficiencias del sistema de salud, como el tiempo de espera excesivo o la escasez de personal, representan mayor riesgo.

De acuerdo a revisiones previas ${ }^{32}$ el abuso verbal es el tipo de VL con mayor prevalencia, lo que puede entenderse por un sesgo de inclusión de la variable. Respecto a la procedencia, a diferencia de lo encontrado en una revisión previa ${ }^{38}$ donde se analizó principalmente violencia interna (colegas y jefes), la mayoría de los estudios seleccionados en esta revisión se focalizan en violencia externa, coincidiendo con lo reportado por Pourshaikhian et al. (2016) en una revisión anterior ${ }^{20}$.

El amplio interés por realizar investigación con personal de enfermería obedece a que se trata de una población que tiene un riesgo mayor de sufrir violencia, lo que se atribuye, a cuestiones relativas al género y a las características del trabajo de cuidado ${ }^{35}$.

De otra parte, los trabajadores de servicios de salud mental también muestran alto riesgo de exposición a VL lo que se vincula con la naturaleza del trabajo como por ejemplo tratar con pacientes bajo efectos de las drogas o con enfermedades psiquiátricas $^{36}$.

Una parte importante de estudios entrega sugerencias organizacionales para reducir o mitigar el fenómeno, como plantean Pourshaikhian et al. es necesario establecer directrices y capacitación para los funcionarios respecto a las que leyes de protección del personal y educar a los usuarios para reducir y controlar el maltrato laboral ${ }^{20}$.

Entre las limitaciones de este estudio se encuentran: la búsqueda se realizó sólo en inglés y español, excluyendo la producción en portugués. Además, no fue posible comparar los hallazgos respecto a la composición de la muestra con revisiones anteriores ya que éstas fueron realizadas mayoritariamente en servicios específicos. Finalmente, la limitación del lugar de trabajo al sector salud y los criterios para la inclusión en la revisión restringen la generalización de los hallazgos a otras poblaciones.

A pesar de que esta revisión advierte respecto de las múltiples consecuencias de la VL y demues- tra claramente sus implicancias para la eficacia de las organizaciones de salud, no se encontraron estudios con muestras nacionales de trabajadores de la salud en Latinoamérica que estimen su prevalencia y den cuenta de la relación entre el fenómeno, sus consecuencias y los factores facilitadores de su surgimiento. Además, la mayoría de los estudios en Latinoamérica se concentran en acoso psicológico ${ }^{4,34}$ siendo que la VL es un fenómeno con múltiples manifestaciones. Finalmente, un desafío importante para la investigación sobre VL es la realización de estudios de carácter mixto que, por una parte, permitan acceder a mayor profundidad en la comprensión del fenómeno mediante las experiencias subjetivas de quienes trabajan en salud y, por otra parte, establezcan prevalencias en esta población, estimen la magnitud de sus consecuencias y propongan mecanismos oportunos y viables para el afrontamiento del fenómeno.

Agradecimientos: Este artículo fue realizado en el marco del Proyecto FONDECYT Regular 2017 N $^{\circ}$ 1170239: "Prevalencia, formas de manifestación y factores facilitadores de la VL y sus efectos en la salud mental en población asalariada en Chile atendiendo desigualdades sociales y de género" el cual cuenta con financiamiento de CONICYT.

\section{Referencias}

1. OIT (2003). La Violencia en el trabajo. Educación Obrera 2003/2004. Disponible en: http://www.ilo.org/ wcmsp5/groups/public/---ed_dialogue/---actrav/documents/publication/wcms_117581.pdf [Consultado el 15 de septiembre de 2016].

2. Einarsen S. Harassment and bullying at work: a review of the Scandinavian approach. Aggression and Violent Behavior 2000; 5 (4): 379-401. Disponible en: http://www.sciencedirect.com/science/article/pii/ S1359178998000433 [Consultado el 2 de octubre de 2017).

3. Hogh A, Viitasara E. A systematic review of longitudinal studies of nonfatal workplace violence. European Journal of Work and Organizational Psychology 2007; 14 (3): 291-313. Disponible en: https://www.researchgate. net/publication/247515795_A_systematic_review_of_ longitudinal_studies_of_nonfatal_workplace_violence [Consultado el 15 de octubre de 2016]. 
4. Ansoleaga E, Gómez-Rubio C, Mauro A. Violencia laboral en América Latina: una revisión de la evidencia científica. Rev Arg de Psiquiat 2015; XXVI: 444-52. Disponible en: https://www.researchgate.net/publication/291161742_Violencia_laboral_en_America_Latina_una_revision_de_la_evidencia_cientifica [Consultado el 10 de octubre de 2016].

5. Magnavita N. Workplace Violence and Occupational Stress in Healthcare Workers: A Chicken-andEgg Situation Results of a 6-Year Follow-up Study. Journal of Nursing Scholarship 2014; 46 (5): 366-76 Disponible en: https://www.researchgate.net/publication/261804059_Workplace_Violence_and_Occupational_Stress_in_Healthcare_Workers_A_Chicken-andEgg_Situation-Results_of_a_6-Year_Follow-up_Study [Consultado el 10 de agosto de 2016].

6. Magnavita N, Fileni A, Pescarini L, Magnavita G. Violence against radiologists. I: prevalence and preventive measures. Radiología Médica 2012; 117 (6): 1019-33. Disponible en: https://link.springer.com/ article/10.1007\%2Fs11547-012-0825-7. [Consultado el 10 de agosto de 2016].

7. Cavanaugh C, Campbell J, Messing JT. A Longitudinal Study of the Impact of Cumulative Violence Victimization on Comorbid Posttraumatic Stress and Depression Among Female Nurses and Nursing Personnel. WorkplaceHealth \& Safety 2014; 62 (6): 224-32. Disponible en: https://www.ncbi.nlm.nih.gov/pubmed/24971817 [Consultado el 8 de agosto de 2016].

8. Arnetz JE, Aranyos D, Ager J, Upfal MJ. Development and Application of a Population-Based System for Workplace Violence Surveillance in Hospitals. American Journal of Industrial Medicine 2011; 54 (12): 925-34. Disponible en: https://www.ncbi.nlm.nih.gov/ pubmed/21739469 [Consultado el 7 de agosto de 2016].

9. Itzhaki M, Peles-Bortz A, Kostisky H, Barnoy D, Filshtinsky V, Bluvstein I. Exposure of mental health nurses to violence associated with job stress, life satisfaction, staff resilience, and post-traumatic growth. International Journal of Mental Health Nursing 2015; 24 (5): 403-12. Disponible en: https://www.ncbi.nlm.nih.gov/ pubmed/26257307 [Consultado el 13 de agosto de 2016].

10. Magnavita N, Heponiemi T. Violence towards health care workers in a Public Health Care Facility in Italy: a repeated cross-sectional study. BmcHealthServicesResearch 2012; 12: 9. Disponible en: https://bmchealthservres.biomedcentral.com/articles/10.1186/1472-696312-108 [Consultado el 10 de agosto de 2016].

11. Bernaldo-de Quiros M, Labrador F, Piccini M, Gómez A, Cedeira J. Violencia laboral en emergencias extra- hospitalarias, una revisión sistemática y líneas de intervención psicológica. Revista Clínica y Salud 25 (2014). Disponible en: http://www.redalyc.org/pdf/1806/Resumenes/Resumen_180630186003_1.pdf. [Consultado el 12 de enero de 2017].

12. Baby M, Glue P, Carlyle D. 'Violence is Not Part of Our Job': A Thematic Analysis of Psychiatric Mental Health Nurses' Experiences of Patient Assaults from a New Zealand Perspective. Issues in Mental Health Nursing 2014; 35 (9): 647-55. Disponible en: https://www.ncbi. nlm.nih.gov/pubmed/25162186. [Consultado el 13 de septiembre de 2016].

13. Dement JM, Lipscomb HJ, Schoenfisch AL, Pompeii LA. Impact of hospital Type II violent events: Use of psychotropic drugs and mental health services. American Journal of Industrial Medicine 2014; 57 (6): 627-39. Disponible en: https://www.ncbi.nlm.nih. gov/pubmed/24526348 [Consultado el 10 de agosto de 2016].

14. Giorgi G, Mikayo A, Arenas A, Shoss M, León-Pérez J. Exploring Personal and Organizational Determinants of Worplace Bullying and its prevalence in a Japanese Sample. Psychology of Violence 2013; 3 (2): 185-97. Disponible en: https://www.researchgate.net/publication/256772191_Exploring_Personal_and_Organizational_Determinants_of_Workplace_Bullying_and_Its_ Prevalence_in_a_Japanese_Sample [Consultado el 15 de noviembre de 2016].

15. Rugulies R, Madsen I, Hjarsbech P, Hogh A, Borg $\mathrm{V}$, Carneiro I, et al. Bullying at work and onset of a major depressive episode among Danish female elder care workers. Scand J Work Environ Health 2012; 38 (3): 218-27. Disponible en: https://www.jstor.org/ stable/41508887?seq=1\#page_scan_tab_contents. [Consultado el 13 de diciembre de 2016].

16. Pien LC, Chen DR, Chen CJ, Liang KM, Cheng YW. Associations of neighborhood-level workplace violence with workers' mental distress problems: a multilevel analysis of Taiwanese employees. J Occup Health 2015; 57 (6): 555-64. Disponible en: https://www.ncbi.nlm. nih.gov/pubmed/26423828. [Consultado el 17 de agosto de 2016].

17. Einarsen S, Nielsen MB. Workplace bullying as an antecedent of mental health problems: a five-year prospective and representative study. Int Arch Occup Environ Health 2015; 88 (2): 131-42. Disponible en: https:// link.springer.com/article/10.1007/s00420-014-0944-7 [Consultado el 27 de diciembre de 2016].

18. Rodwell J, Demir D. Psychological consequences of bullying for hospital and aged care nurses. International Nursing Review 2012; 59 (4): 539-46. Disponible 
en: https://www.ncbi.nlm.nih.gov/pubmed/23134139. [Consultado el 15 de agosto de 2016].

19. De Puy J, Romain-Glassey N, Gut M, Wild P, Mangin P, Danuser B. Erratum to: Clinically assessed consequences of workplace physical violence. Int Arch Occup Environ Health 2015; 88: 225. Disponible en: https://www.researchgate.net/publication/263095498_Erratum_to_Clinically_assessed_consequences_of_workplace_physical_violence. [Consultado el 20 de agosto de 2016].

20. Pourshaikhian M, Abolghasem H, Aryankhesal A, Khorasani-Zavareh D, Barati A. A Systematic Literature Review: Workplace Violence Against Emergency Medical Services Personnel. Arch Trauma Res 2016; 5 (1): e28734. Disponible en: https://www.ncbi.nlm.nih. gov/pubmed/27169096 [Consultado el 23 de diciembre de 2016].

21. Lanctôt N, Guay S. The aftermath of workplace violence among healthcare workers: A systematic literature review of the consequences. Aggression and Violent Behavior 2014; 19: 492-501. Disponible en: https://www. researchgate.net/publication/264936956_The_aftermath_of_workplace_violence_among_healthcare_workers_A_systematic_literature_review_of_the_consequences. [Consultado el 20 de diciembre de 2016].

22. Beltrán O. Revisiones sistemáticas de la literatura. Rev. Colombiana de Gastroenterología 20 (1) 2005. Disponible en: http://www.scielo.org.co/pdf/rcg/v20n1/ v20n1a09.pdf. [Consultado el 27 de diciembre de 2016].

23. Berelson. Content Analysis in Communication Researches. Glencoe III, Free Press. 1952. En: López F. El análisis de contenido como método de investigación. (2002). Revista de Educación 2002; 4: 167-79. Disponible en: http://rabida.uhu.es/dspace/bitstream/ handle/10272/1912/b15150434.pdf [Consultado el 2 de octubre de 2017].

24. Gálvez A. Enfermería Basada en la Evidencia. Cómo incorporar la investigación a la práctica de los cuidados. Granada: Fundación Index. 2001.

25. Einarsen S, Raknes BI, Matthiesen SB, Helleøy OH. Mobbing ogHarde Personkonflikter: Helsefarligsamspillpåarbeidsplassen [Bullying and harsh personal conflicts: Health damaging interactions at work] Bergen, Norway: Sigma Forlag, 1994.

26. Arnetz JE. The Violent Incident Form (VIF): a practical instrument for the registration of violent incidents in the health care workplace. Work \& Stress 1998; 12: 1728. Disponible en: http://www.tandfonline.com/doi/ abs/10.1080/02678379808256846. [Consultado el 17 de diciembre de 2016].

27. OSHA. Guidelines for preventing WPV for health care and social service workers, OSHA 3148-01R. Washing- ton, DC: Occupational Safety and Health Administration. 2004. Disponible en: www.osha.gov/Publications/ osha3148.pdf [Consultado el 21 de octubre de 2016].

28. Ridenour M, Lanza M, Hendricks S, Hartley D, Rierdan $\mathrm{J}$, Zeiss R, et al. Incidence and risk factors of workplace violence on psychiatric staff. Work- a Journal of Prevention Assessment \& Rehabilitation 2015; 51 (1): 19-28. Disponible en: https://www.ncbi.nlm.nih.gov/pmc/ articles/PMC4674794/. [Consultado el 10 de noviembre de 2016].

29. Sabri B, St Vil N, Campbell J, Fitzgerald S, Kub J, Agnew J. Racial and Ethnic Differences in Factors Related to Workplace Violence Victimization. Western Journal of Nursing Research 2015; 37 (2): 180-96. Disponible en: https://www.ncbi.nlm.nih.gov/pmc/articles/ PMC4169764/ [Consultado el 24 de enero de 2017].

30. Wood S, Braeken J, Niven K. Discrimination and Well-Being in Organizations: Testing the Differential Power and Organizational Justice Theories of Workplace Aggression. J Bus Ethics 2013; 115. 617-34. Disponible en: https://www.researchgate.net/publication/257541836_ Discrimination_and_Well-Being_in_Organizations_ Testing_the_Differential_Power_and_Organizational_ Justice_Theories_of_Workplace_Aggression [Consultado el 21 de diciembre de 2016].

31. Taylor J, Rew L. A systematic review of the literature workplace violence in the emergency department. Journal of Clinical Nursing 2010; 20: 1072-85. Disponible en: https://www.ncbi.nlm.nih.gov/pubmed/20846214 [Consultado el 12 de enero de 2017].

32. Guay S, Goncalves J, Jarvis J. Verbal violence in the workplace according to victims sex- a systematic review of the literature. Agression and Violent Behavior 2014; 19: 572-8. Disponible en: https://www.researchgate.net/ publication/264899972_Verbal_violence_in_the_workplace_according_to_victims'_sex_-_a_systematic_review_of_the_literature [Consultado el 15 de noviembre de 2016].

33. Topa G, Depolo M, Morales JF. Acoso laboral: metaanálisis y modelo integrador de sus antecedentes y consecuencias. Psicothema 2007; 19 (1): 88-94. Disponible en: http://www.redalyc.org/articulo.oa?id=72719113 [Consultado el 23 de octubre de 2016].

34. Toro JP, Gómez-Rubio C. Facilitadores de la Violencia Laboral: Una revisión de la evidencia científica en América Latina. Ciencia y Trabajo, año 18, número 56, mayo- agosto 2016. Disponible en: http://www. scielo.cl/scielo.php?script=sci_arttext\&pid=S071824492016000200006 [Consultado el 15 de enero de 2017].

35. Wlosko M, Ros C. Violencia laboral en el sector salud: 
abordajes conceptuales y resultados de investigación en personal de enfermería en Argentina. En: Ansoleaga E, Artaza O, Suárez J, editores. Personas que cuidan personas: dimensión humana y trabajo en salud. Santiago: OPS/OMS; 2012. p. 211-57.

36. Travetto C, Daciuk N, Fernández S, Ortiz P, Mastandue- no R, Prats M, et al. Agresiones hacia profesionales en el ámbito de la salud. Rev. Panam. Salud Pública, 2015; 38 (4): 307-15. Disponible en: http://www.scielosp.org/ scielo.php?pid=S1020-49892015000900007\&script $=$ sci_abstract\&tlng=es. [Consultado el 12 de diciembre de 2016]. 